\title{
Asplenia e hiposplenia en pediatría. Prevención de sepsis bacteriana fulminante
}

\author{
Fernanda Cofré y José Cofré
}

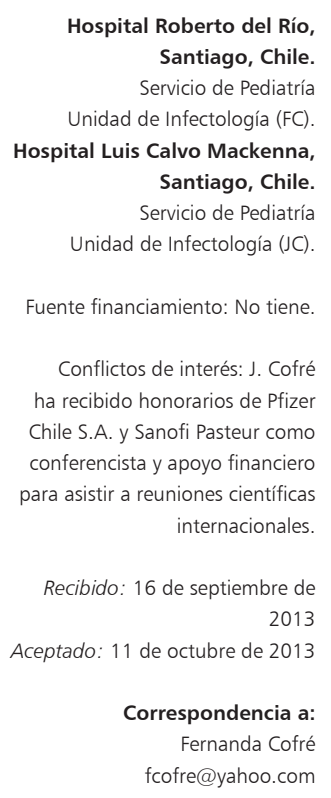

\section{Introducción}

$\mathrm{E}$ 1 bazo es el mayor órgano linfoide del cuerpo humano y en él convergen funciones y estructuras del sistema circulatorio, retículoendotelial e inmu$\mathrm{ne}^{1}$. Las funciones esplénicas clásicamente se dividen en cuatro categorías²: filtración, reservorio, hematopoyesis e inmunidad.

En términos de función inmunológica el bazo participa de la inmunidad innata y la inmunidad adquirida con funciones que son comunes a otros órganos y funciones que le son propias. Entre las funciones inmunes inespecíficas del bazo está la producción de mediadores de la inmunidad involucrados en la eliminación de bacterias lo que le hace tener un rol fundamental en la prevención de la sepsis bacteriana ${ }^{2,3}$.

El rol esplénico en la prevención de sepsis bacteriana demostrado por la sepsis bacteriana fulminante-SBF post esplenectomía, (en inglés denominada overwhelming post splenectomy infection = OPSI), está dado por la capacidad del bazo de filtrar y fagocitar las bacterias desde la sangre de un hospedero no inmune, remover material o partículas extrañas a él (parásitos, eritrocitos, etc.) y la producción precoz de opsoninas: IgM antígeno específicas, componentes del complemento, properdina y tufsina ${ }^{1,4,5}$.

\section{Definiciones}

Asplenia: ausencia de bazo, que puede ser secundaria a una remoción quirúrgica o, en forma menos frecuente, congénita ${ }^{6}$.
Hiposplenia: desorden adquirido en que hay un mal funcionamiento del bazo, asociado a múltiples enfermedades y generalmente acompañado de una disminución en el tamaño esplénico ${ }^{6,7}$.

\section{Diagnóstico}

El diagnóstico de asplenia o disfunción esplénica está fundamentalmente basado en la visualización de anormalidades específicas de los eritrocitos en el frotis sanguíneo (cuerpos de Howell Jolly), en el diagnóstico de imágenes y en la evaluación de la función de filtración esplénica mediante métodos radioisotópicos ${ }^{1,6,8}$. Estos últimos tienen uso clínico limitado por su alto costo y dificultades técnicas.

La detección de cuerpos de Howell-Jolly en forma persistente en el frotis sanguíneo es un método útil como tamizaje para detectar una asplenia aunque su especificidad y sensibilidad son discutidas, especialmente en estados de hipoesplenismo ${ }^{6}$. Raramente se pueden observar si el bazo no es francamente disfuncional o está sobre-exigido por otras funciones fagocíticas como la hemólisis extravascular ${ }^{1,8}$.

La cuantificación de eritrocitos con pitts en su membrana es utilizado como estándar de oro para el diagnóstico de disfunción esplénica en la mayoría de los estudios porque es un método simple, cuantitativo, reproducible y se correlaciona con el volumen esplénico pero requiere de un microscopio de interferencia de fases lo que limita su uso clínico ${ }^{6,8}$.

El hallazgo de un bazo pequeño o ausente durante la 
realización de imágenes abdominales requiere de un estudio dirigido para certificar su función, a cualquier edad, especialmente en pacientes portadores de cardiopatías congénitas complejas con isomerismo o con antecedentes familiares de asplenia congénita ${ }^{9,10}$.

Tabla 1. Condiciones en las cuales es recomendable, en algún momento de la evolución, considerar esplenectomía

Enfermedades hematológicas
- Anemias hemolíticas congénitas
- Enfermedades hematológicas adquiridas mediadas por inmunidad
- Púrpura trombocitopénico idiopático
- Anemia hemolítica autoinmune
- Hiperesplenismo
- Enfermedad de Gaucher
- Talasemia mayor
Esplenomegalia congestiva
Indicaciones quirúrgicas
- Trauma esplénico
- Quistes y tumores esplénicos
- Alivio mecánico de los síntomas originados por la esplenomegalia

\section{Tabla 2. Causas de hipoesplenismo funcional}

\begin{tabular}{l} 
Hematológicas \\
- Hemoglobinopatías \\
- Histiocitosis \\
- Anemia de Fanconi \\
Autoinmunes \\
- Vasculitis con infarto esplénico \\
- Lupus eritematoso sistémico \\
- Artritis reumatoide \\
- Enfermedad de Graves \\
- Síndrome de Sjögren \\
- Poliarteritis nodosa \\
Enfermedades crónicas gastrointestinales \\
- Enfermedad celiaca \\
- Colitis ulcerosa \\
- Linfangectasia intestinal \\
- Enfermedad de Whipple \\
Enfermedades hepáticas \\
- Hepatitis crónica activa \\
- Cirrosis \\
- Infiltración hepática \\
- Enfermedades de depósito: Gaucher, Niemann-Pick \\
- Amiloidosis con compromiso esplénico \\
- Sarcoidosis con compromiso esplénico \\
Vasculares \\
- Oclusión de la arteria esplénica \\
- Trombosis de la vena esplénica \\
Misceláneas \\
- Infección por VIH \\
- Enfermedad de injerto contra hospedero post trasplante de pre- \\
- Trrsores hematopoyéticos \\
- Nutrición parenteral total \\
- Irradiación esplénica \\
\hline
\end{tabular}

Para evaluar la función esplénica, el método de elección es la cintigrafía con Tc 99 ya que permite evaluar todas las facetas de la función esplénica y la presencia de eventuales bazos supernumerarios y/o ectópicos funcionales ${ }^{6,8}$.

En función de lo anterior frente a un paciente con antecedentes de presencia de cuerpos de Howell-Jolly en el hemograma, ser portador de cardiopatía congénita compleja o tener antecedentes familiares de asplenia congénita, es imperativo realizar una ecografía abdominal con búsqueda dirigida del bazo y, ante el hallazgo de un bazo pequeño o su ausencia, realizar un cintigrama para evaluar su función.

\section{Causas}

Quirúrgicas. La principal indicación de esplenectomía en pediatría es los estados hiperesplénicos que dan cuenta de más de $50 \%$ de las esplenectomías, mientras que el trauma abdominal es indicación de 10 a $30 \%$ de las esplenectomías ${ }^{3,4}$.

En la Tabla 1 se encuentran las condiciones clínicas en las cuales se puede llegar a considerar la esplenectomía quirúrgica.

Asplenia congénita. Puede darse como un hallazgo aislado o como parte de un síndrome que frecuentemente va asociado a anormalidades cardiovasculares (síndrome de Ivemark $)^{3,8}$. Sobre el $90 \%$ de los casos se asocia a cardiopatías congénitas complejas, dentro de las que se describen dos grandes grupos: asplenia asociada con isomerismo derecho que se presenta con pulmones trilobulares bilaterales y situs inversus abdominal, y polisplenia asociada con isomerismo izquierdo que se presenta con pulmones bilobulares bilaterales y heterotaxia abdominal ${ }^{11}$.

En la Tabla 2 se enumeran las condiciones clínicas en las cuales se puede llegar a desarrollar una hiposplenia/ asplenia funcional.

\section{Sepsis bacteriana fulminante}

Se refiere al cuadro clínico de septicemia, meningitis o neumonía fulminante gatillada por bacterias ricas en cápsula, principalmente Streptococcus pneumoniae, Neisseria meningitidis y Haemophilus influenzae tipo b en pacientes asplénicos o hiposplénicos ${ }^{6,12,13}$.

El pródromo generalmente es leve, consistente con un cuadro febril, en que el sello está dado por un deterioro clínico en el curso de horas, asociado a compromiso hemodinámico, coagulación intravascular diseminada y falla multiorgánica que no responde a medidas habituales y que puede simular una meningococcemia fulminante ${ }^{1,5}$.

La incidencia real de SBF es desconocida pero las estimaciones derivadas de la observación de cohortes 
muestran una incidencia anual de 0,23 a $0,42 \%$ post esplenectomía con un riesgo de 5\% a lo largo de la vida ${ }^{4,5,12,14}$. La letalidad global es de 50 a $70 \%$ y puede descender a $10 \%$ con un tratamiento médico agresivo y precoz ${ }^{5}$; la mayor tasa de mortalidad se da en el grupo de pacientes bajo 2 años de edad ${ }^{3}$.

$\mathrm{El}$ riesgo de infecciones graves o muerte es mayor en los primeros tres años post esplenectomía y el riesgo de una $2^{\mathrm{a}}$ o $3^{\mathrm{a}}$ infección es particularmente elevado en aquellos pacientes con una primera infección grave y dentro de los primeros seis meses posteriores a ella ${ }^{15,16}$. Esta condición de riesgo no se extingue a lo largo de la vida aunque se reduce a un mínimo según transcurren los años.

La esplenectomía realizada por trauma tiene una menor incidencia de SBF que aquella secundaria a enfermedades hematológicas autoinmunes, probablemente porque estas últimas llevan asociado una terapia inmunosupresora que aumenta el riesgo de infecciones graves per se.

La asplenia congénita es una condición mayor predisponente para SBF y en la mayoría de los casos presenta un patrón de herencia autosómico dominante ${ }^{5}$ con una incidencia real desconocida para SBF.

\section{Microbiología}

Streptococcus pneumoniae. Ha sido identificado como la causa de 50 a $90 \%$ de los casos de SBF en pacientes con asplenia, con una mortalidad global que va de 50 a $70 \%{ }^{7,14,16-18}$. Los serotipos recuperados de pacientes asplénicos son los mismos que predominan y producen patología en los pacientes sin asplenia ${ }^{18,19}$. Se reporta internacionalmente que los serotipos más frecuentemente aislados en asplénicos son 6B, 23F, 18C y 19A. Diferentes serotipos pueden ser responsables de diferentes infecciones neumocóccicas invasoras en un mismo paciente ${ }^{18}$.

Desde el año 2004, Chile participa en la Red de Vigilancia de Agentes Bacterianos Responsables de Neumonía y Meningitis (Sistema Regional de Vigilancia-SIREVA) a través del Instituto de Salud Pública (ISP), laboratorio de referencia nacional. Durante el período 2007-2012 se ha podido observar que los serotipos más frecuentes de $S$. pneumoniae que producen enfermedad neumocóccica invasora (ENI) en Chile son los serotipos 14 (18\%), 1 $(11,8 \%)$ y $7 \mathrm{~F}(6,9 \%)$, seguidos por los serotipos $3,6 \mathrm{~A}$ y $6 \mathrm{~B}$, lo que da una cobertura teórica para las vacunas conjugadas 10 valente y 13 valente de 59,4 y 73,6\%, respectivamente, en la población general y de 69 y 84,1\%, respectivamente, para los niños bajo 2 años de edad y no guarda relación con lo descrito por la literatura científica internacional $^{20}$. En el caso de la vacuna polisacárida 23 valente el único serotipo de los más frecuentes que no estaría cubierto sería el 6A y esto daría racionalidad a la conducta de vacunar primero con una vacuna conjugada para inducir inmunidad de memoria y luego con la vacuna polisacárida 23 valente, para mejorar la cobertura respecto a los serotipos más prevalentes en nuestro país.

Neisseria meningitidis. Es una causa importante de $\mathrm{SBF}$ en pacientes asplénicos después de $S$. pneumoniae.

Haemophilus influenzae tipo b. También se ha descrito como un importante agente productor de SBF en pacientes asplénicos pero actualmente tiene menor frecuencia dado la vacunación universal contra $H$. influenzae tipo b, iniciada en nuestro país en el año 1996.

Capnocytophaga canimorsus ha sido descrita como otro microorganismo causal de SBF, en individuos con el antecedente de mordedura por perro o gato ${ }^{4,7,14}$.

También se describe una mayor susceptibilidad a la malaria y babesiosis, entidades que no están presentes en nuestro país pero que se deberían considerar en caso de viajes a zonas endémicas ${ }^{4,5,12}$.

\section{Prevención de la sepsis bacteriana fulminante en pacientes asplénicos/ hiposplénicos}

\section{Recomendaciones básicas}

- El médico especialista (cardiólogo, hematólogo, etc.) responsable de la atención de los pacientes que padezcan una de las afecciones asociadas a asplenia/ hiposplenia, debe evaluar en forma oportuna, precoz, y si es necesario, periódicamente, la funcionalidad del bazo.

- Es altamente recomendable que los pacientes asplénicos usen un elemento identificatorio (medalla o pulsera) que advierta a terceros su condición de asplénico.

- El médico general, o de servicios de urgencia debe tener un claro concepto, ante la consulta de un paciente asplénico/hiposplénico por un cuadro febril, del potencial desarrollo de una SBF.

\section{Educación}

La educación a los pacientes, sus familiares y médicos tratantes debe estar enfocada en la comprensión del mayor riesgo de infecciones bacterianas invasoras en este grupo de pacientes haciendo énfasis en la premura de la consulta en caso de fiebre o deterioro del estado general y el inicio de un tratamiento antimicrobiano precoz para evitar el desarrollo de $\mathrm{SBF}^{1,4,7,14}$. Para esto último se recomienda alguna de las siguientes dos conductas:

- Antimicrobianos stand-by: amoxicilina de inicio inmediato frente a cualquier enfermedad febril mientras se concreta la consulta precoz en un servicio de urgencia ${ }^{4,14}$. 
- Una vez en el servicio de urgencia, la administración de antimicrobianos parenterales con cobertura sobre los agentes productores de SBF (cefalosporinas de tercera generación), previa toma de hemocultivos y proceder a su hospitalización. En estos casos, la positividad de los hemocultivos es usualmente de $95 \% \%^{4,5,14}$.

No existe consenso en si una u otra estrategia es la más recomendada $y$, en general, se utilizan ambas en forma simultánea hasta que exista certeza de si el paciente se encuentra cursando una SBF o no. Esto naturalmente lleva al sobre-tratamiento de episodios febriles pero disminuye la morbi-mortalidad asociada a SBF.

\section{Inmunización}

La inmunización programática de un paciente asplénico/hiposplénico debe ser con las vacunas de rutina correspondientes por edad; en ella está incluida al día de hoy la vacunación del lactante contra $H$. influenzae tipo b y $S$. pneumoniae. El ser asplénicos no es una contraindicación para recibir las vacunas vivas contenidas en el Programa Nacional de Inmunizaciones (PNI), ${ }^{7}$.

Vacunas especiales son las vacunas meningocóccicas conjugadas tetravalentes e influenza. Aquellos pacientes que por edad no recibieron las vacunas anti-neumocóccicas y anti $H$. influenzae tipo b, deben recibirlas, en forma independiente de su edad.

\section{Vacunación anti-neumocóccica}

\section{Conceptos generales}

En caso de una esplenectomía electiva, es recomendable completar el esquema primario de vacunación anti-neumocóccica por lo menos con dos semanas previas al acto quirúrgico, dado que la respuesta inmune es mejor cuando el bazo aún está presente ${ }^{7,14}$. Frente a una esplenectomía de urgencia, se ha demostrado en pacientes adultos en quienes se indicó vacuna neumocóccica polisacárida, que los mejores niveles de anticuerpos antineumocóccicos se alcanzan administrando la vacuna 14 días post esplenectomía; pero, en aquellos casos en que existe incertidumbre de si la vacuna será administrada en forma ambulatoria, se recomienda efectuar esta vacunación previa al alta hospitalaria ${ }^{3,7}$.

En un estudio realizado en Finlandia se observó que 84 a 100\% de los pacientes asplénicos logra un aumento significativo de las concentraciones de anticuerpos específicos contenidos en la vacuna llegando a títulos protectores luego de aplicar el esquema primario de vacuna anti-neumocóccica conjugada 7 valente, en forma independiente de la causa de la asplenia o esplenectomía y de la edad ${ }^{17}$.

En pacientes inmunocomprometidos, la respuesta a vacunas no conjugadas (polisacárida) es sub-óptima y las concentraciones de anticuerpos decaen rápidamente en el tiempo (24 a 32\% durante el primer año post inmunización) lo que no se observa al administrar vacunas conjugadas $^{21}$.

\section{Esquema de vacunación}

Pacientes con asplenia congénita. La vacuna antineumocóccica conjugada 10 ó 13 valente se indica en todos los niños desde los 2 meses de vida según el esquema programático universal chileno a los 2, 4 y 12 meses, a partir de enero de 2011. Es recomendable que los pacientes asplénicos reciban un esquema de cuatro dosis (2, 4, 6 y 12 meses) como se indica en la Tabla 3, de manera de otorgarles la máxima protección desde el primer año de vida ${ }^{22,23}$. Ello se sustenta en que el esquema de 3 dosis (2, 4 y 12 meses), vigente en el PNI chileno y en diversas otras naciones, no estimula en forma satisfactoria la respuesta específica contra los serotipos 3, 6B y $23 \mathrm{~F}$,

\begin{tabular}{|c|c|c|c|c|c|}
\hline Edad & $\begin{array}{l}\text { Neumocóccica conjugada } \\
\qquad 10 \text { ó } 13 \text { valente }\end{array}$ & $\begin{array}{l}\text { Neumocóccica polisacári- } \\
\text { da } 23 \text { valente }\end{array}$ & $\begin{array}{c}\text { Vacuna } \\
\text { H. influenzae b }\end{array}$ & $\begin{array}{l}\text { Meningocóccica conjugada } \\
\text { tetravalente } \mathrm{ACWY}^{*}\end{array}$ & Influenza \\
\hline 2 meses & $x$ & & $x$ & $X^{*}$ & \\
\hline 4 meses & $x$ & & $x$ & $X^{*}$ & \\
\hline 6 meses & $x$ & & $x$ & $X^{*}$ & X (anual) \\
\hline 9 meses & & & & $X^{*}$ & \\
\hline 12 meses & $x$ & & & $X^{*}$ & \\
\hline 18 meses & & & $x$ & & \\
\hline 24 meses & & $x$ & & & \\
\hline 7 años & & $x$ & & & \\
\hline
\end{tabular}


fenómeno que ha sido observado tanto con las vacunas 7,11 y 13 valentes como con la vacuna 10 valente vi-27. $^{27}$

No hay estudios que hagan referencia a la eficacia protectora comparativa de vacunas conjugadas 10 valente y 13 valente en población pediátrica general ni tampoco en este grupo específico de pacientes. En una postura teórica, se podría recomendar, cuando sea posible, la administración de la vacuna conjugada 13 valente en cuatro dosis por su potencial mayor cobertura contra serotipos causantes de enfermedad invasora.

La vacuna polisacárida debe administrarse en una dosis, a continuación, a los 24 meses de edad ${ }^{16}$.

La re-vacunación con vacuna anti-neumocóccica polisacárida a los 3-5 años de la primera dosis se recomienda para niños con asplenia que tienen menos de 10 años de edad y que ya recibieron una dosis de vacuna polisacárida y para adultos y niños sobre 10 años de edad que recibieron su esquema de vacunación primaria por lo menos 5 años antes ${ }^{16,17}$. Una tercera dosis de vacuna polisacárida no se recomienda en forma rutinaria ${ }^{16,17}$.

Pacientes con hiposplenia o asplenia adquirida (quirúrgica). Deben recibir dos dosis de vacuna anti-neumocóccica conjugada 10 valente o 13 valente espaciadas por 60 días y luego, una dosis de vacuna neumocócica polisacárida 23 valente, dos meses más tarde.

\section{Determinación de anticuerpos anti-neumocóccicos}

La real utilidad de la medición de anticuerpos antineumocóccicos no está clara y su evaluación puede dar una falsa sensación de seguridad si el paciente alcanzó niveles considerados protectores tras un esquema de vacunación completo ${ }^{14}$. La medición de anticuerpos alcanza a 10 serotipos, los principales en forma universal, pero deja fuera serotipos no vaccinales potencialmente causantes de SBF; en la actualidad, ello representa aproximadamente $26-40 \%$ de las infecciones invasoras serotipificadas en Chile por el ISP como se expresara más arriba. Por otra parte, la introducción de vacunas conjugadas en la población infantil, podría favorecer la aparición de serotipos no contenidos en dichas vacunas, acentuando la futilidad de medir la respuesta inmune a la vacunación.

\section{Vacunación anti-H. influenzae tipo b}

En Chile se aplica esta vacuna desde el año 1996, de manera que, en teoría, la población de pacientes pediátricos está enteramente vacunada. No se ha construido evidencia que haga recomendable revacunar a los niños en general, ni a pacientes asplénicos/hiposplénicos en especial. Para $H$. influenzae tipo b se alcanzan altos niveles de anticuerpos capsulares (anti poli-ribo-fosfato) en $97 \%$ de los pacientes asplénicos con la primera dosis del esquema primario de vacunación ${ }^{14,21}$.

Además, las infecciones causadas por $H$. influenzae tipo $b$ han disminuido universalmente a una mínima incidencia en los países que incorporaron esta vacuna al PNI.

\section{Vacunación anti-meningocóccica}

La respuesta inmunológica a la vacuna anti-meningocóccica depende del motivo de la asplenia y del tipo de vacuna utilizada. La inmunización con vacuna polisacárida capsular no se recomienda en niños asplénicos debido a que este tipo de vacuna otorga una protección de corta duración (1-2 años) y no existe un producto en base a polisacárido capsular contra el serogrupo B, el más prevalente en nuestro país hasta el año $2011^{28,29}$. Con la introducción de las vacunas conjugadas contra los serogrupos A, C, W e Y, capaces de inducir inmunidad a largo plazo, la vacunación de pacientes asplénicos contra $N$. meningitidis es ahora una recomendación. Una dosis única de vacuna anti-meningocóccica conjugada tetravalente resulta en una inadecuada respuesta de anticuerpos en 33\% de los pacientes asplénicos, en forma independiente de su edad; una segunda dosis lleva a un aumento adicional en el título de anticuerpos y su avidez, lo que sugiere un beneficio adicional en pacientes asplénicos ${ }^{16,28}$. Es necesario estar atento a la vigilancia realizada por el ISP respecto al potencial cambio en los serogrupos circulantes y a la próxima licencia de vacunas anti meningocóccicas B para su uso clínico.

\section{Vacunación anti-influenza}

La vacuna contra influenza está recomendada en forma anual desde los 6 meses de edad a todo paciente asplénico/hiposplénico dado la asociación existente entre la infección por influenza y la sobreinfección por S. pneumoniae ${ }^{7,14,30}$.

\section{Profilaxis antimicrobiana}

Como se expresara anteriormente, la vacunación por sí sola no es suficiente protección para estos pacientes y están descritos casos de SBF en pacientes adecuadamente vacunados ${ }^{31}$. Por eso, actualmente se recomienda profilaxis antimicrobiana diaria para enfermedad neumocóccica invasora en todos los pacientes asplénicos e hiposplénicos, especialmente antes de cumplir 5 años de edad, independiente de su estado de inmunización ${ }^{4,7,16}$.

Datos de estudios en pacientes con anemia de células falciformes sugieren que aquellos pacientes asplénicos que no han tenido infecciones neumocóccicas invasoras graves y que han recibido su esquema completo de inmunización anti-neumocóccica pueden suspender la profilaxis antimicrobiana a los 5 años de edad. La necesidad de la profilaxis en asplenia por otras causas es desconocida; algunos expertos sugieren mantener la profilaxis anti-neumocóccica de por vida dado que 5\% de los paciente asplénicos padecerán una SBF a lo largo de su vida ${ }^{4,7,14-16,18}$. 
El antimicrobiano recomendado usualmente es penicilina V oral (no disponible en Chile) aunque otras guías usan amoxicilina ${ }^{3,4,7,16}$. En caso de alergia documentada a penicilina son alternativas válidas en la literatura internacional eritromicina y cotrimoxazol $1^{4,7,16}$ pero considerando que en nuestro país existe actualmente $30 \%$ de resistencia in vitro a eritromicina en cepas invasoras de S. pneumoniae ${ }^{20}$ podrían ser una alternativa excepcional el uso de cefadroxilo o cloranfenicol (Tabla 4). Las inyecciones mensuales de penicilina benzatina logran concentraciones séricas de penicilina inferiores a las requeridas ${ }^{3,7}$ por lo que actualmente se prefiere el uso de profilaxis vía oral.

\section{Resumen}

El bazo tiene la capacidad intrínseca de prevenir infecciones bacterianas invasoras dado su habilidad de filtrar y fagocitar las bacterias de la sangre, retirar partículas o elementos ajenos a ella y la producción precoz de opsoninas. La ausencia anatómica del bazo generalmente

\begin{tabular}{|c|c|c|}
\hline Fármaco & Edad & Dosis diaria \\
\hline Penicilina $\vee$ oral & $\begin{array}{l}<\text { de } 5 \text { años } \\
\geq \text { de } 5 \text { años }\end{array}$ & $\begin{array}{l}125 \mathrm{mg} \mathrm{c} / 12 \mathrm{~h} \\
250 \mathrm{mg} \mathrm{c} / 12 \mathrm{~h}\end{array}$ \\
\hline Amoxicilina & $\begin{array}{l}<\text { de } 5 \text { años } \\
\geq \text { de } 5 \text { años }\end{array}$ & $\begin{array}{l}20 \mathrm{mg} / \mathrm{kg} \text { (fraccionar } \mathrm{c} / 12 \mathrm{~h} \text { ) } \\
250 \mathrm{mg} \mathrm{c} / 12 \mathrm{~h}\end{array}$ \\
\hline Cotrimoxazol & 40/8 mg/kg/día de sulfa/trimetoprim & \\
\hline Cefadroxilo & $30 \mathrm{mg} / \mathrm{kg} /$ (fraccionar cada $12 \mathrm{~h}$ ) & \\
\hline Cloranfenicol & $20-30 \mathrm{mg} / \mathrm{kg}$ (fraccionar cada $12 \mathrm{~h}$ ) & \\
\hline
\end{tabular}

es secundaria a su remoción quirúrgica y también puede ser congénita. Streptococcus pneumoniae es el principal agente responsable de los episodios de infecciones bacterianas fulminantes y conlleva a una alta mortalidad en este grupo de pacientes. Se presenta una exhaustiva revisión de la evidencia y recomendaciones actualmente disponibles en la literatura para la prevención de infecciones invasoras en este grupo específico de pacientes.

\section{Referencias bibliográficas}

1.- Sumaraju V, Smith L, Smith S. Infectious complications in asplenic hosts. Infect Dis Clin North Am 2001; 15 (2): 551-65.

2.- Cadili A, De Gara A. Complications of splenectomy. Am J Med 2008; 121: 371-5.

3.- Price V, Blanchette V, Ford-Jones L. The prevention and management of infections in children with asplenia or hyposplenia. Infect Dis Clin North Am 2007; 21: 697-710.

4.- Price V, Dutta S, Blanchette V, Butchart S, Kirby M, Langer J, et al. The prevention and treatment on bacterial infections in children with asplenia or hyposplenia: Practice considerations at the Hospital for Sick Children, Toronto. Pediatr Blood Cancer 2006; 46: 597-603.

5.- Morgan T, Tomich E. Overwhelming postsplenectomy infection (OPSI): a case report and review of the literature. J Emerg Med 2012; 43 (4): 758-63.

6.- Di Sabatino A, Carsetti R, Corazza G. Post-splenectomy and hiposplenic states. Lancet 2011; 378: 86-97.

7.- Vicente M, Moreno-Pérez D, Núñez E, Rivera M, Hagerman A, García F. Manejo de niños con asplenia/hipoesplenia. Asociación Española de Pediatría 2008. http:// dc372.4shared.com/doc/CHeWsEYD/preview. $\mathrm{html}$

8.- Tarantino G, Savastano S, Capone D, Colao A. Spleen: a new role for an old player?. World J Gastroenterol 2011; 17 (33): 3776-84.

9.- Mahlaoui N, Minard-Colin V, Picard C, Bolze A, Ku CL, Tournilhac O, et al. Isolated congenital asplenia: A French nationwide retrospective survey of 20 cases. J Pediatr 2011; 158: 142-8.

10.- Bertrán K, Donoso A, Cruces P, Díaz F, Arriagada D. Asplenia congénita y purpura fulminans neumocóccico en paciente pediátrico. Reporte de caso con necropsia y revisión del tema. Rev Chilena Infectol 2009; 26 (1): 55-9.

11.- Dyke M, Martin R, Berry P. Septicaemia and adrenal haemorrhage in congenital asplenia. Arch Dis Child 1991; 66: 636-7.

12.- Jones P, Leder K, Woolley I, Cameron P, Cheng A, Spelman D. Postsplenectomy infection. Strategies for prevention in general practice. Austr Fam Physician 2010; 39 (6): 383-6.

13.- Waghorn D. Overwhelming infection in asplenic patients: current best practice preventive measures are not being followed. J Clin Pathol 2001: 54: 214-8.

14.- Davidson R, Wall R. Prevention and management of infections in patients without a spleen. Clin Microbiol Infect 2001; 7: 657-60.

15.- Kyaw M, Holmes E, Toolis F, Wayne B, Chalmers J, Jones I, et al. Evaluation of severe infection and survival after splenectomy. Am J Med 2006; 119 (3): 276.e1-276.e7.

16.- American Academy of Pediatrics. Immunization in special clinical circumstances. In Pickering $\mathrm{L}$, Baker C, Kimberlin D, Long S, eds. Red Book: 2012 Report of the Committee on Infectious Disease. Elk Grove Villege, IL: American Academy of Pediatrics; 2012: 74-90.

17.- Mikoluc B, Kayhty H, Bernatowska E, Motkowski R. Immune response to the 7-valent pneumococcal conjugate vaccine in 30 asplenic children. Eur J Clin Microbiol Infect Dis 2008; 27: 923-8.

18.- Schutze G, Mason E, Barson W. Invasive pneumococcal infections in children with asplenia. Pediatr Infect Dis J 2002; 21: 278-82.

19.- Hausdorff W, Bryant J, Paradiso P, Siber G. Which pneumococcal serogrups cause the most invasive disease: implications for conjugated vaccine formulation and use. Clin Infect Dis 2000; 30: 100-21.

20.- Boletín Instituto de Salud Pública de Chile. Vigilancia de Enfermedad Invasora Streptococcus pneumoniae Chile, 2007-2012. Boletín ISP 2013; 3 (3): 1-23. http://www.isp.cl/ sites/default/files/Boletin\%20ENI.pdf

21.- Meerveld-Eggink A, de Weerdt O, van Velzen-Blad H, Biesma D, Rijkers G. Response to conjugated pneumococcal anh Haemophilus influenzae type $\mathrm{b}$ vaccines in asplenic patients. Vaccine 2011; 29: 675-80.

22.- Resolution No. 06/10-1 ACIP 2010. Advisory Committee on Immunization Practices Vaccines for Children Program Vaccines to Prevent Pneumococcal Disease. http://www.cdc.gov/ vaccines/recs/acip/downloads/agenda-jun10.pdf

23.- Centers for Disease Control and Prevention. Use of 13-valent pneumococcal conjugate vaccine and 23-valent pneumococcal polysaccharide vaccine among children aged 6-18 years with immunocompromising conditions: Recommendations of the Advisory Committee on Immunization Practices (ACIP). MMWR Morb Mortal Wkly Rep June 28, 2013; 62; 25: 521-4. 
24.- Rennels M B, Edwards K M, Keyserling H L. Safety and immunogenicity of heptavalent pneumococcal vaccine conjugated to CRM197 in United States infants. Pediatrics 1998; 101 (4 pt 1): 604-11.

25.- Givon Lavi N, Greenberg D, Dagan R. Immunogenicity of alternative regimens of the conjugated 7-valent pneumococcal vaccine: a randomized controlled trial. Pediatr Infect Dis J 2010; 29 (8): 756-62.

26.- Snape M D, Klinger C L, Daniels E D, John T M, Layton H, Rollinson L, et al. Immunogenicity and reactogenicity of a 13 -valent pneumococcal conjugate vaccine administered at 2, 4 and 12 of age: a double blind randomized active-controlled trial. Pediatr Infect Dis J 2010; 29 (12): e80-90.

27.- Sigverdal S, Hogh B, Bergsaker M R,
Skerlikova H, Lomel P, Boris D, et al. Immunogenicity of a 2-dose priming and booster vaccination with the 10 -valent pneumococcal non typeable Haemophilus influenzae protein D conjugate vaccine. Pediatr Infect Dis J 2009; 28 (10): e276-82.

28.- Meerveld-Eggink A, de Weerdt O, de Voer R, Berbers G, van Velzen-Blad H, Vlaminckx B, et al. Impaired antibody response to conjugated meningococcal serogroup $\mathrm{C}$ vaccine in asplenic patients. Eur J Clin Microbiol Dis 2011; 30: 611-8.

29.- Boletín Instituto de Salud Pública de Chile. Vigilancia de Laboratorio de Enfermedad Invasora por Neisseria meningitidis. Laboratorio de Referencia 2006-2011. Boletín ISP 2012; 2 (2): 1-18. http://www.ispchile.cl/sites/files/ Boletin\%20Neisseria\%20Meningitidis\%20 26\%20marzo.pdf
30.- Langley J, Dodds L, Fell D, Langley R Pneumococcal and influenza immunization in asplenic persons: a retrospective populationbased cohort study 1990-2002. BMC Infect Dis 2010; 10: 219.

31.- Forstner C, Plefka S, Tobudic S, Winkler H, Burgmann K, Burgmann H. Effectiveness and immunogenicity of pneumococcal vaccination in splenectomised and functionally asplenic patients. Vaccine 2012; 30: 5449-52.

*En forma adicional a las referencias bibliográficas anotadas, se consultó sobre esta materia con dos reconocidos expertos: Dr. Stanley Plotkin Profesor de la Universidad de Pensilvania y Asesor en vacunas de Sanofi Pasteur y Dr. Ron Dagan, Director de la Unidad de Enfermedades Infecciosas del Departamento de Pediatría de Universidad de Soroka, Beer-Sheva, Israel. 